\title{
Impact of Physicochemical Profiling for Rational Approach on Drug Discovery
}

\author{
Shoko Nakashima, ${ }^{* \dagger}$ Katsuhiko Yamamoto, ${ }^{\dagger}$ Yuta Arai, ${ }^{\dagger}$ and Yukihiro Ikeda ${ }^{\dagger}$ \\ Drug Metabolism and Pharmacokinetics Research Laboratories, Pharmaceutical Research Division, Takeda \\ Pharmaceutical Company, Ltd.; 2-26-1 Muraoka-higashi, Fujisawa, Kanagawa 251-8555, Japan. \\ Received June 4, 2013; accepted September 4, 2013
}

Solid-state characterization plays a vital role in lead optimization and candidate selection with the appropriate physicochemical properties for proper oral dosage formulation. Aqueous solubility is an important parameter in the successful development of oral dosage formulation since poor aqueous solubility limits absorption. In this study, we summarized an efficient approach using a small amount of sample for solidstate characterization, including thermodynamic solubility, which is defined as physicochemical profiling. By using the physicochemical profiling results of 75 anti-cancer drugs and clinical candidates, we examined the relationship between thermodynamic solubility and molecular structural parameters and assessed the effects of thermodynamic solubility on pharmacokinetic profile for rational soluble drug design. The $\log D_{\mathrm{pH} 7.4}$, aromatic ring count, and hydrogen bond count were good indicators for predicting sparingly soluble compounds that increase the lattice energy because of $\pi-\pi$ stacking and hydrogen bonds, resulting in lowered thermodynamic solubility. The level of thermodynamic solubility in simulated intestinal fluid ( $\mathrm{pH}$ 6.8) in the presence and absence of bile acid, which is required for minimal acceptable bioavailability $(>30 \%)$, was $1 \mu \mathrm{g} /$ $\mathrm{mL}$ and $10 \mu \mathrm{g} / \mathrm{mL}$, respectively. Physicochemical profiling, which includes thermodynamic solubility considering its solid-state properties, contributes to rational lead optimization and efficient candidate selection for drug development.

Key words physicochemistry; thermodynamic solubility; kinetic solubility; lipophilicity; bioavailability

The most common administration route of drugs entering the commercial market is oral administration due to its ease and convenience in terms of drug delivery. ${ }^{1,2)}$ Solubility is a crucial parameter that affects oral absorption as well as the permeability of solid dosage forms. ${ }^{3,4)}$ Low aqueous solubility results in poor absorption and variability in the drug plasma concentration, which results in an attrition rate and high cost of drug development. ${ }^{4,5)}$

Many studies have reported on the importance of the solid form due to its effects on solubility and stability. ${ }^{6-9)}$ In general, the difference in solubility between the polymorphic or crystalline forms and the amorphous forms was less than 10 times or up to several hundred times, respectively. ${ }^{10)}$ Thus, an evaluation of the solubility taking into account its solid-state properties is essential during drug development.

Within the pharmaceutical industry, there are 2 different types of solubility measurements: kinetic solubility and thermodynamic solubility. ${ }^{5)}$ Kinetic solubility is a measurement of solubility that dilutes a highly concentrated compound organic solution into an aqueous solution. In contrast, thermodynamic solubility is equilibrium solubility of solid crystalline material in an aqueous solution. Although kinetic solubility is performed in high throughput and is preferably measured at the early discovery stage, kinetic solubility is sometimes overestimated because the precipitates are amorphous or in a meta-stable form, according to Ostwald's rule, because of fast-precipitation when the drug stock solution is added to the aqueous solution or by shaking. ${ }^{11,12)}$ Thus, the thermodynamic

\footnotetext{
The authors declare no conflict of interest.

$\dagger$ Present address: Physicochemistry \& Preformulation, Research \& Development, Analytical Development Laboratories, CMC Center, Takeda Pharmaceutical Company, Ltd.; 2-26-1 Muraoka-higashi, Fujisawa, Kanagawa 251-8555, Japan.
}

solubility measurement on the basis of the solid form is a critical element for efficient and successful development in the solid dosage formulation.

During lead optimization and candidate selection, we evaluated the thermodynamic solubility measurement on the basis of the solid form using the following 3 main analyses: (1) powder X-ray diffraction (PXRD), (2) thermogravimetry (TG) and differential scanning calorimetry (DSC) analyses and (3) thermodynamic solubility measured by the shake-flask method, which is defined as physicochemical profiling. Physicochemical profiling enables the assessment of thermodynamic solubility by considering the solid-state properties in the early drug development stage. It also contributes to the batch analysis and evaluation of drug-likeness in drug discovery and is a key indicator of whether the solid-state properties of the drug are sufficient for development into an oral dosage form.

A decade has passed since the Rule of 5, which defined the molecular structural parameters for drug-like compounds using approximately 2000 candidates or late-stage trial compounds, according to Lipinski. ${ }^{5,13)}$ Given that the Rule of 5 was easy-to-use, it has been widely used for the assessment of drug-likeness. Variations of the Rule of 5, such as Rule of $3^{14)}$ and the Rule of $4,{ }^{15)}$ have been proposed and many studies have recently attempted to analyze the relationship between molecular structural parameters and solubility. ${ }^{16,17)}$ These studies have gained much attention in the physicochemical field. The molecular structural parameters, such as the molecular weight, lipophilicity, polar surface area, and aromatic ring, have been reported as solubility parameters. ${ }^{3,18-20)}$ Although a few correlation studies between the molecular structural parameters and thermodynamic solubility have been reported, they used thermodynamic solubility without consideration of the crystallinity and crystal form. ${ }^{21-23)}$ Thermodynamic solubility without consideration of the crystallinity and crystal 
form was not suitable for the relationship analysis between the molecular structural parameters and solubility since thermodynamic solubility is affected by the crystallinity and crystal form. In addition, there has been no study about the factor creating the discrepancy between thermodynamic solubility and kinetic solubility and the correlation between thermodynamic solubility and oral absorption.

In this study, we present physicochemical profiling, which uses medium throughput ${ }^{24)}$ and a systematic approach with a small amount of drugs in the early discovery stage. Using the physicochemical profiling results of 75 anti-cancer drugs and clinical candidates, we examined the correlation between molecular structural parameters and the thermodynamic solubility and discussed the effects of solubility on oral absorption. In addition, we discuss factors that cause differences between the kinetic and thermodynamic solubility. This is the first work which analyzes the relationship between thermodynamic solubility based on the crystallinity and crystal form and the molecular structural parameters, investigates the discrepancy between thermodynamic solubility and kinetic solubility and studies the correlation between thermodynamic solubility and oral absorption.

\section{Experimental}

Materials Seventy-five anti-cancer drugs and clinical candidates were selected as tool compounds in this study because most of them were poorly soluble and a wide range of solubility was covered. Their physicochemical profiling results and the pharmacokinetic (PK) parameters in rats are presented in Table 1. Some solids existed in hydrates or solvates, amorphous revealed by PXRD and the thermal analysis. These data were eliminated from the relationship analysis between thermodynamic solubility and the molecular structural parameters since they were not suitable due to the considerable influence of the solid state properties on solubility. The inequality sign of kinetic solubility data is sometimes used due to the dependency of the maximum solubility values on the molecular weight because the drug solution with a given concentration is diluted with aqueous media in the kinetic solubility measurement. To investigate the effect of thermodynamic solubility on oral absorption and oral absorption rate, the rat oral bioavailability and mean absorption time (MAT) data of the compounds with low permeability, large clearance or large volumes of distribution was not available for the analysis of the relationship between solubility and PK data.

All of the Takeda compounds were synthesized in the Medicinal Chemistry Research Laboratories and Chemical Development Laboratories at Takeda Pharmaceutical Co., Ltd. (TPC). Other anti-cancer drugs and clinical candidates were purchased from Wako Pure Chemical Industries, Ltd. (Osaka, Japan), Sigma-Aldrich (St. Louis, MO, U.S.A.), Tokyo Chemical Industry Co., Ltd. (Tokyo, Japan) and Tocris Bioscience (Bristol, U.K.).

\section{Physicochemical Profiling}

PXRD PXRD patterns were collected using a RINT U1timaIV powder X-ray diffractometer (Rigaku Corp., Tokyo, Japan) with $\mathrm{Cu} K \alpha$ radiation, generated at $40 \mathrm{kV}$ and $50 \mathrm{~mA}$. The drug substances were placed on a silicone plate at room temperature. The plates were then inserted into a 48-position Automatic Sample Changer, which enables an automatic con- tinuous measurement of the 48 samples. The data were collected from 2 to $35^{\circ}(2 \theta)$ at a step size of $0.02^{\circ}$ and scanning speed of $6 \% \mathrm{~min}$.

Crystallinity Calculation After subtracting the background pattern of the silicon plate from all of the PXRD patterns of the drug sample, the peak intensities of the crystalline $\left(I_{\mathrm{c}}\right)$ and non-crystalline $\left(I_{\mathrm{a}}\right)$ fractions were integrated from the baseline collection using Herman's method. ${ }^{25,26)}$ The crystallinity was calculated using the following equation:

$$
\text { Crystallinity }=I_{\mathrm{c}} \times 100 /\left(I_{\mathrm{c}}+I_{\mathrm{a}}\right) \times 100
$$

To avoid any variability derived from daily and personal errors, the crystallinity was calculated using an autocrystallinity calculation software (Rigaku, Tokyo, Japan). ${ }^{26,27)}$

Thermal Analysis TG was performed using a TGA/ DSC1 system (Mettler-Toledo International Inc., Greifensee, Switzerland). A TG curve was obtained in an open aluminum pan using ca. $0.60 \mathrm{mg}$ of the drug substance and a heating rate of $5^{\circ} \mathrm{C} / \mathrm{min}$ under a nitrogen gas flow at $40 \mathrm{~mL} / \mathrm{min}$. The DSC was measured using a DSC1 system (Mettler-Toledo International Inc., Greifensee, Switzerland). A DSC thermogram was obtained in a crimped aluminum pan using $c a .0 .25 \mathrm{mg}$ of drug substance and a heating rate of $5^{\circ} \mathrm{C} / \mathrm{min}$ under a nitrogen flow at $20 \mathrm{~mL} / \mathrm{min}$.

Thermodynamic Solubility Measurement by the ShakeFlask Method To measure the thermodynamic solubility, $0.4 \mathrm{mg}$ of the drug substances was weighed into Thomson filter vials (Chrom Tech, Inc., Minnesota, U.S.A.) containing a $0.45 \mu \mathrm{M}$ polyvinylidene difluoride filter membrane. Four hundred microliters of JP1 ( $\mathrm{pH} 1.2)$, JP2 (pH6.8), and JP2containing $20 \mathrm{~mm}$ sodium glyco-chenodeoxycholate (GCDC), were added to the vials. The vials were incubated at $37^{\circ} \mathrm{C}$ with shaking at $500 \mathrm{rpm}$ for $18 \mathrm{~h}$ and the resulting suspensions were filtrated by compressing the vials. The drug concentration of the filtrates was determined by HPLC.

High Performance Liquid Chromatography (HPLC) Analysis HPLC analysis was conducted using a UHPLC system (Prominence UFLC, Shimadzu Corp., Kyoto, Japan) with a photodiode array detector. A YMC-UltraHT Pro C18 packaged column $(2 \mu \mathrm{m}, 2.0 \times 3.0 \mathrm{~mm}$, YMC Co., Ltd., Kyoto, Japan) was used at $40^{\circ} \mathrm{C}$ at a flow rate of $0.7 \mathrm{~mL} / \mathrm{min}$. The gradient mobile phase consisted of distilled water- $50 \mathrm{~mm}$ ammoniun acetate buffer-acetonitrile $(8: 1: 1, \mathrm{v} / \mathrm{v} / \mathrm{v})$ (A) and $50 \mathrm{~mm}$ ammoniun acetate buffer-acetonitrile (1:9, v/v) (B). The starting mobile phase consisted of $95 \% \mathrm{~A}$ and $5 \% \mathrm{~B}$ for $0.1 \mathrm{~min}$, changed gradually to $5 \% \mathrm{~A}$ and $95 \% \mathrm{~B}$ from 0.1 to $1.6 \mathrm{~min}$, and then returned to the initial condition after $1.3 \mathrm{~min}$ and was re-equilibrated for an additional $0.6 \mathrm{~min}$.

Kinetic Solubility Measurement by the Precipitation Method The kinetic solubility was evaluated by the precipitation method. ${ }^{28,29)}$ The compounds were dissolved in dimethylsulfoxide (DMSO) to yield $10 \mathrm{~mm}$ DMSO stock solutions. To measure the kinetic solubility, a 96-well multi-filter plate (Millipore Corp., MA, U.S.A.) was used. DMSO stock solutions $(5 \mu \mathrm{L})$ were dispensed into a 96-well multi-filter plate, and $245 \mu \mathrm{L}$ of JP1 and JP2 and JP2-containing $20 \mathrm{~mm}$ GCDC were added. The plate was incubated at $25^{\circ} \mathrm{C}$ for $24 \mathrm{~h}$. After filtration, the drug concentration was analyzed using HPLC.

$\log D_{\text {pH 7.4 }}(\log D)$ Determination $\log D$, which is a partition coefficient between 1-octanol and aqueous buffer $\mathrm{pH}$ 7.4, of the compounds was measured using a chromato- 


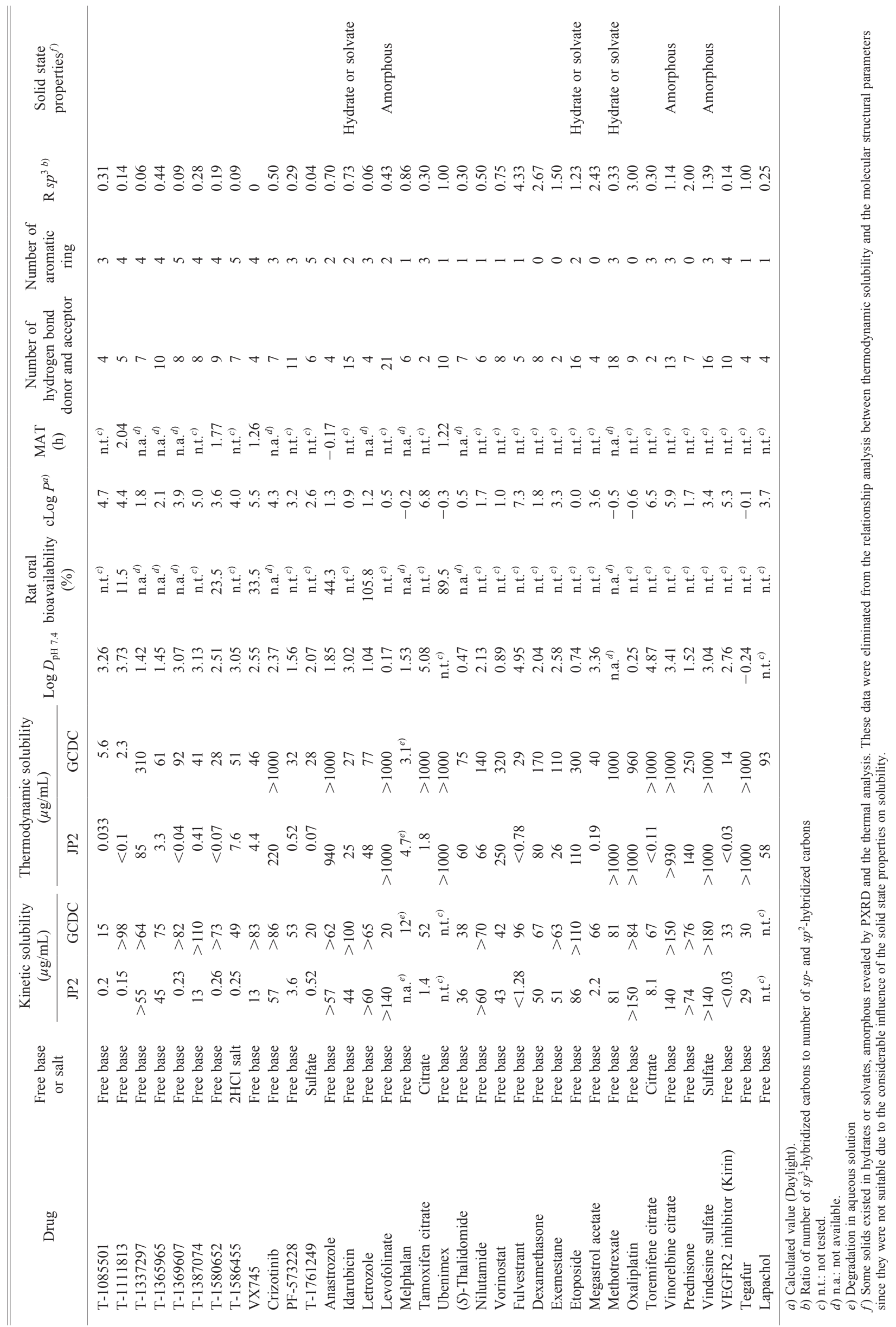




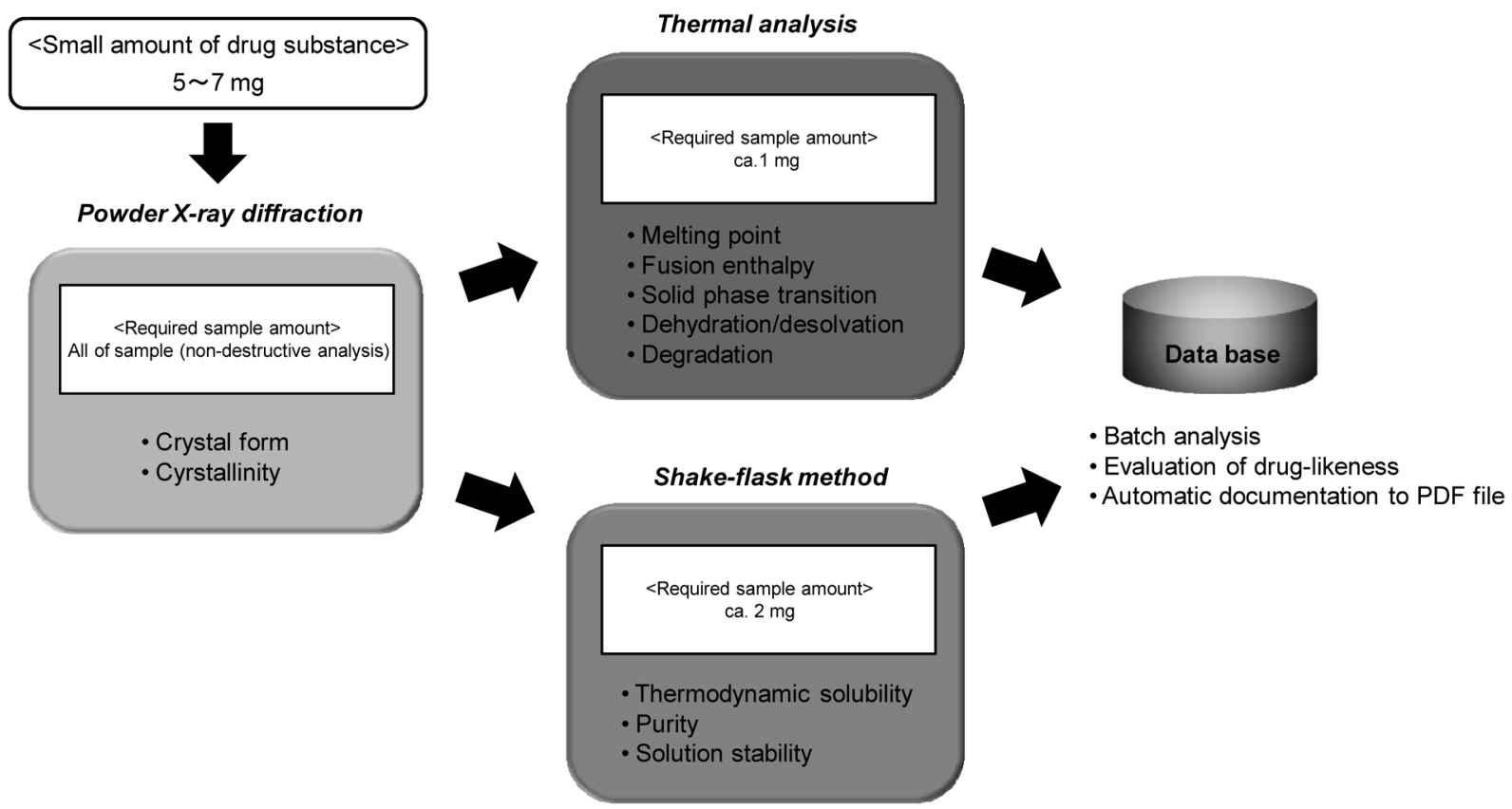

Fig. 1. TPC Workflow for Physicochemical Profiling

graphic method as previously described. ${ }^{30)}$ A Waters Alliance 2795 HPLC system with a 2487 UV-Vis detector was used (Milford, MA, U.S.A.).

Data Visualization and Statistical Analysis A TIBCO spotfire (TIBCO Software Inc., Somerville, MA, U.S.A.) was used to visualize and conduct the statistical analysis.

PK Study in Rats All animal experiments were carried out in accordance with the guidelines of the Institutional Animal Care and Use Committee (Takeda Pharmaceutical Co., Ltd.). The PK parameters were obtained by cassette dosing of the compounds in rats. ${ }^{31,32)}$ Fasted Jcl:SD male rats $(8$ weeks old) were used. Prior to dosing, rats were anesthetized with a femoral vein injection of isoflurane. A mixture of no more than 10 drugs was administered at doses of $0.1 \mathrm{mg} / \mathrm{kg}$ (intravenous or i.v.) and $1 \mathrm{mg} / \mathrm{kg}$ (oral or per os (p.o.)). Blood samples were then collected from the tail vein using a Pasteur pipette with heparin in regular intervals for $8 \mathrm{~h}$. The concentration of all of the plasma samples was determined using the LC-MS/MS method. The HPLC apparatus comprised an SIL-10AHc (Shimadzu, Kyoto, Japan). The analytical column was a Inertsil C8 column $(2.1 \times 33 \mathrm{~mm})$ and the mobile phase consisted of acetonitrile and acetonitrile- $10 \mathrm{~mm}$ aqueous ammonium formate $(15: 85, \mathrm{v} / \mathrm{v})$ with a flow rate of $0.2 \mathrm{~mL} / \mathrm{min}$ in a run time of $8 \mathrm{~min}$. The HPLC apparatus was coupled to a Sciex API 3000 mass spectrometer (MDS SCIEX, Concord, Ontario, Canada), which was operated by an ESI source in the positive mode. Positive ion mode with turbo spray was used in the multiple reaction monitoring.

\section{Results and Discussion}

Takeda Pharmaceutical Co., Ltd. (TPC) Approach for Physicochemical Profiling The investigation of solid-state properties is essential to successful development due to its influences on the chemical and physical properties of the drug including crystallinity, solubility, and stability. ${ }^{8)}$ Physicochemical profiling is employed at TPC via lead optimization using medium throughput screening with a sample amount of $7 \mathrm{mg}$, which provides crucial information on the formulation strategy and the candidate developability. The solid-state characteristics are investigated using analytical techniques such as PXRD and thermal analysis (TG/DSC), and the thermodynamic solubility measurement by the shake-flask method.

The schematic TPC approach for physicochemical profiling is shown in Fig. 1. Physicochemical profiling was performed on an equivalent of $7 \mathrm{mg}$ in the powder state. First, the crystal form and crystallinity were identified by PXRD, enabling rapid and non-destructive analysis. Subsequently, thermal characterization (TG and DSC), which investigates the melting point, fusion enthalpy, solid phase transition, and dehydration/desolvation during heating, and thermodynamic solubility measurement by the shake-flask method using the Thomson filter vials, which are designed to minimize drug adsorption on to the filter components compared to the Mini-uni prep ${ }^{\mathrm{TM}}$ (Whatman PLC, Maidstone, U.K.), were performed. ${ }^{33)}$

Measuring the solubility in gastrointestinal fluids provides a good prediction of the in vivo oral absorption behavior. Although it is well known that a solubility comparison in FaSSIF and FeSSIF can help predict food effects in vivo, ${ }^{34)}$ we used fed-state simulated intestinal fluids such as JP2, which contains $20 \mathrm{~mm}$ GCDC, since GCDC is one of the major components in human bile acid ${ }^{35)}$ and is suitable for HPLC analysis.

Previous reports have described that thermodynamic solubility measurements with the crystal form determination was performed during the late stage candidate development ${ }^{24}$ ) since most compounds obtained at the lead optimization process are amorphous, have low purity or the crystal form may not be characterized due to the large sample consumption and low throughput. However, using our TPC approach to physicochemical profile enables the measurement of the thermodynamic solubility including the crystal form and crystallinity as a medium throughput screening with a small amount of sample at the optimization process, which contributes to 
(a)

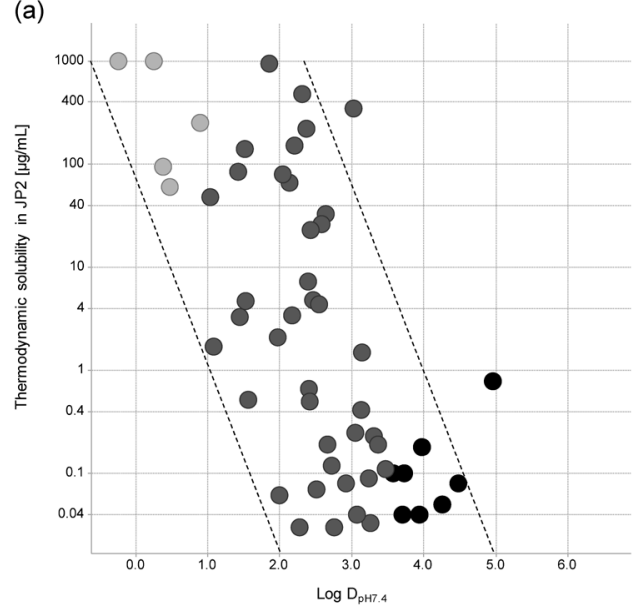

(b)

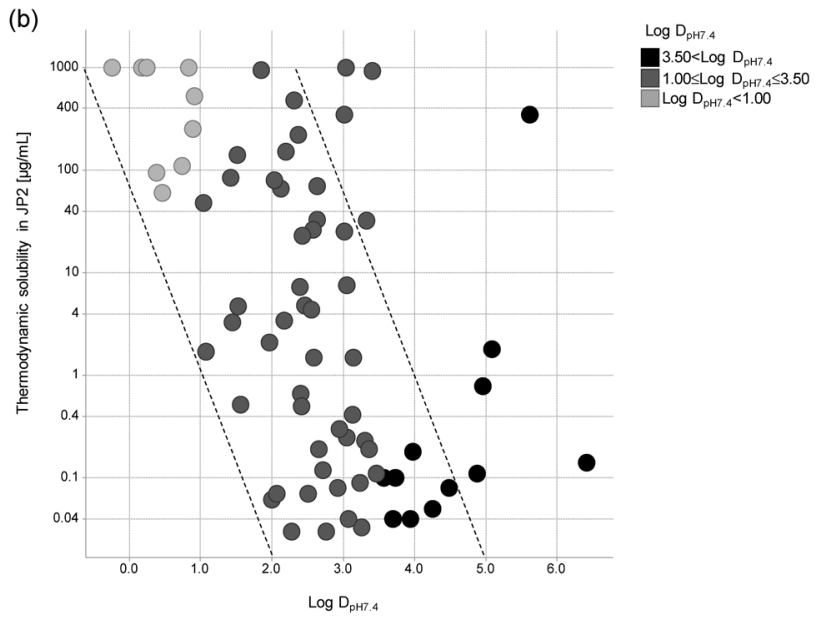

Fig. 2. Correlation between Thermodynamic Solubility and Measured $\log D_{\mathrm{pH} 7.4}$ Value (a); Measured $\log D_{\mathrm{pH} 7.4}$ Value Including the Compounds Whose Solids Were Amorphous and Solvate or Hydrate (b)

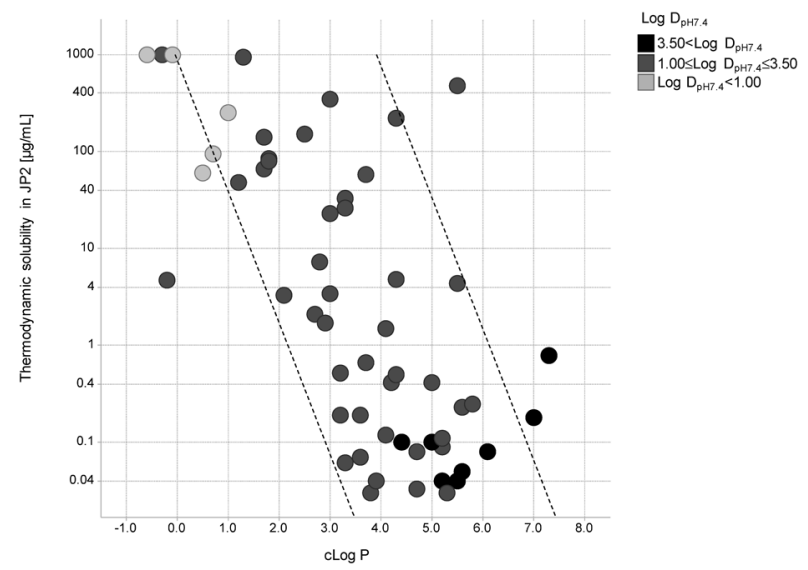

Fig. 3. Correlation between Thermodynamic Solubility and Calculated cLog $P$ Value

the lead optimization and candidate selection for oral dosage formulation.

Contribution of Lipophilicity to Aqueous Solubility Lipophilicity is a well-known parameter that affects aqueous solubility and permeability. Previous studies have shown that increases in lipophilicity results in a high risk for poor oral absorption and variation in drug plasma concentration due to low aqueous solubility. ${ }^{7,8)}$ Although the importance of lipophilicity has been proposed using the relationship between lipophilicity and solubility, they have been mainly used in kinetic solubility data. ${ }^{36,37)}$ In this study, we examined the effect of lipophilicity on thermodynamic solubility. The $\log D$ was used as a measure for lipophilicity of the compounds. The relationship between thermodynamic solubility and $\log D$ in this data set is illustrated in Fig. 2a. Based on the lipophilicity of $\log D$ the thermodynamic solubility may have different propensities. The compounds were categorized in $\log D$ as follows, (1) compounds with $\log D<1$, (2) compounds with $1 \leq \log D \leq 3.5$, and (3) compounds with $\log D>3.5$. All of the compounds of category (1) showed a high solubility ( $>40 \mu \mathrm{g} / \mathrm{mL}$ ). In addition, the category (3) compounds were poorly soluble $(<1 \mu \mathrm{g} / \mathrm{mL})$. Furthermore, the compounds of category (2) showed low-, medium- and high-solubility and a

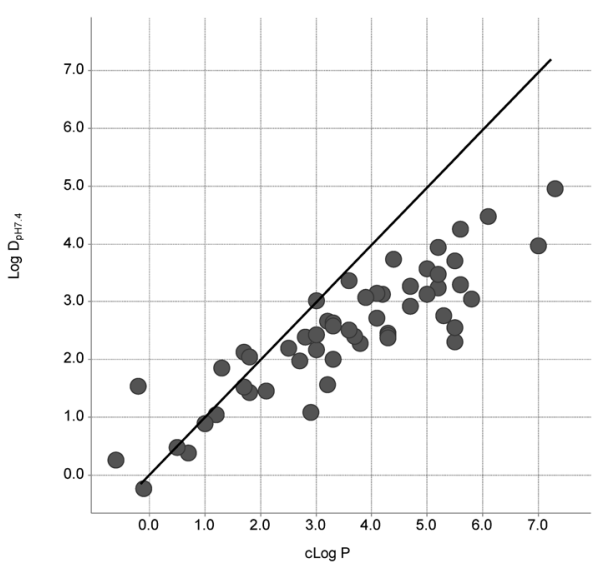

Fig. 4. Correlation between Measured $\log D_{\mathrm{pH} 7.4}$ Value and Calculated cLog $P$ Value

lipophilicity-independent tendency.

Figure $2 \mathrm{~b}$ showed the relationship between thermodynamic solubility and lipophilicity. No correlation was observed including the compounds whose solids were amorphous and solvate or hydrate. These findings indicated that it is important to measure thermodynamic solubility based on the crystal form and crystallinity by TPC approach.

The $\log D$ was particularly useful in providing a more clear differentiation among the poorly soluble compounds compared to $\operatorname{cLog} P{ }^{37)}$ We investigated the differences in the tendency between $\log D$ and $\operatorname{cLog} P$ calculated by $\operatorname{cLog} P$ ver. 4.82 (Daylight Chemical Information Systems Inc.) on thermodynamic solubility. The $\log D$ enhanced the discrimination between the soluble and poorly soluble compounds compared to $\operatorname{cLog} P$ (Fig. 3). The relationship between $\log D$ and $\operatorname{cLog} P$ was shown in Fig. 4. $\log D$ was different from $\operatorname{cLog} P$ because $\operatorname{cLog} P$ is a constant for the molecule under its neutral form and $\log D$ takes into account all neutral and charged forms of the molecule. The poor correlation between $\operatorname{cLog} P$ and thermodynamic solubility was observed among the compounds with the large discrepancy between cLog $P$ and $\log D$ since they have dissociable groups. These results suggested that $\log D$, but not $\operatorname{cLog} P$, was a good indicator of 
poorly soluble compounds.

These findings suggested that $\log D$ is very useful to identify compounds with very high (category (1)) and very poor (category (3)) solubility, in which the solvation process plays an important role in thermodynamic solubility. In these two regions, thermodynamic solubility depends on lipophilicity due to the very smaller value of the enthalpy change of the crystal lattice breaking in the dissolution process than the enthalpy change of the solvation.

The prediction regarding aqueous solubility based on lipophilicity was challenging because most of the drugs have a $\log D$ between 1 and 3.5. ${ }^{38)}$ It may be presumed that the breakage of the crystal lattice can affect thermodynamic solubility of the compounds with a $\log D$ between 1 and 3.5 since the enthalpy change of the solvation in the dissolution process compared to the enthalpy change of the crystal lattice breaking.

These results suggested that thermodynamic solubility among these compounds is considerably affected by the crystal packing and other molecular structural parameters, except for lipophilicity, may contribute to thermodynamic solubility.

Molecular Structural Parameters That Affect on Thermodynamic Solubility Recent studies have indicated that the combination of $\log D$ values together with the aromatic ring count in a given molecule may be a powerful tool to predict kinetic solubility. ${ }^{22}$ We also examined the relationship between thermodynamic solubility in JP2, and the sum of the $\log D$ and aromatic ring count in category (2) compounds (Fig. 5). Compounds with a sum of $\log D$ and aromatic ring count of less than 5 were very soluble (Fig. 5). In addition, we analyzed the relationship between thermodynamic solubility and other molecular structural parameters aside from the aromatic ring count. In the partial region of $\log D$ between 1 and 3.5 , there is an increasing sum in the hydrogen bond donor and acceptor counts, resulting in a lowered thermodynamic solubility (Fig. 6).

These findings suggested that the aromatic ring and the sum of the hydrogen bond donor and acceptor could contribute to thermodynamic solubility of $\log D$ ranging from 1 to 3.5 .

Molecular Structural Parameters That Affect on the Discrepancy between Kinetic Solubility and Thermodynamic Solubility A comparison between kinetic solubility and thermodynamic solubility in this study was performed. The relationship between thermodynamic solubility and kinet- ic solubility in the JP2 and JP2-containing GCDC, respectively, are presented in Figs. 7a and b. The line in both of these figures indicates the $y=x$ relationship (variable $x$ and $y$ means thermodynamic solubility and kinetic solubility, respectively). In the compounds indicated above the line, the thermodynamic solubility was lower than the kinetic solubility. Thus, there were variable extents in the discrepancy between kinetic solubility and thermodynamic solubility. In particular, the discrepancy in JP2 was much larger in the range of $\log D$ from

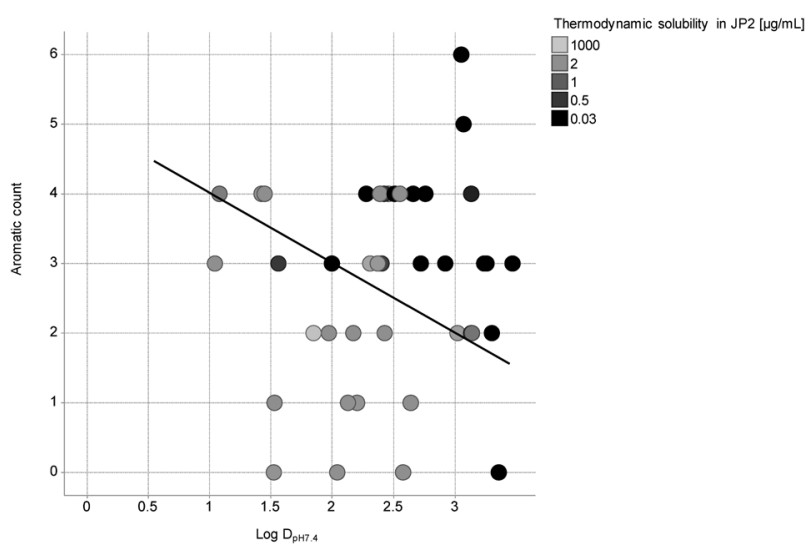

Fig. 5. Distribution of Thermodynamic Solubility in Measured $\log D_{\mathrm{pH} 7.4}$ and Aromatic Ring Count

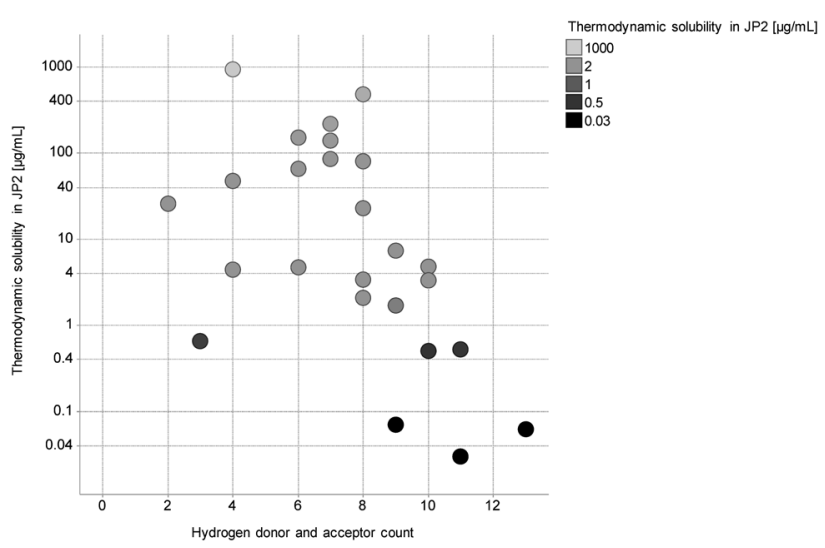

Fig. 6. Distribution of Thermodynamic Solubility in Hydrogen Bond Donor and Acceptor Count
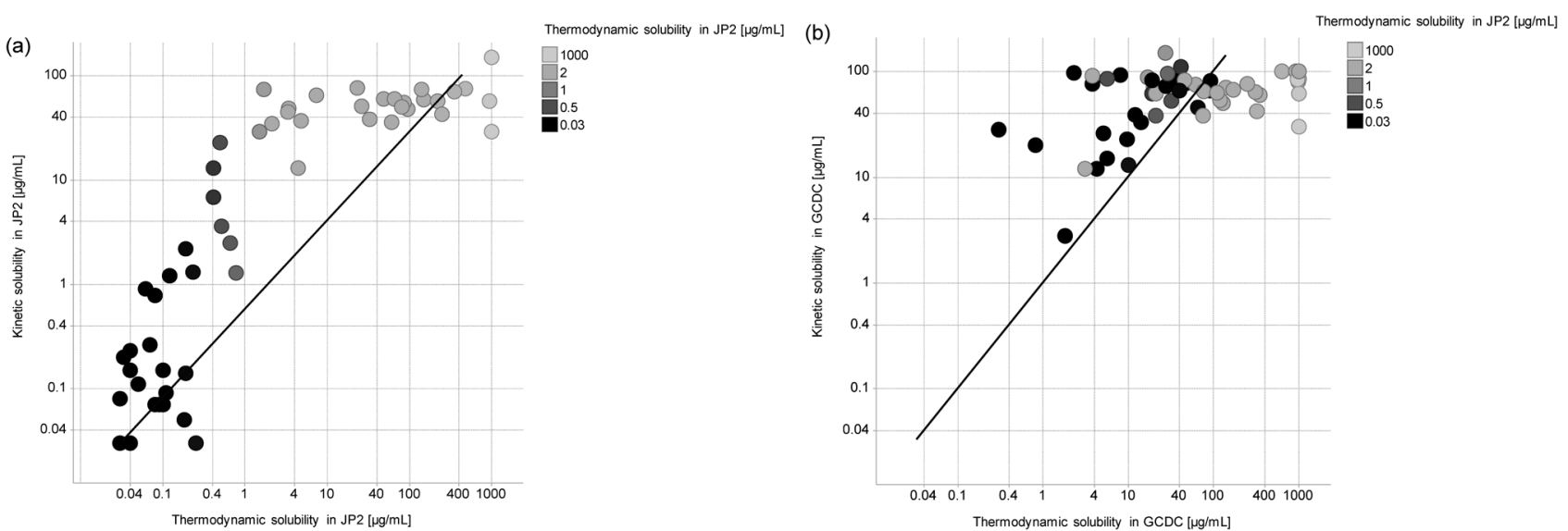

Fig. 7. Difference between Kinetic Solubility and Thermodynamic Solubility in JP2 (a); JP2-Containing GCDC (b) 
1 to 3.5 (Fig. 8a). In contrast, no discrepancy was observed in the compounds from categories (1) and (3). The discrepancy was larger as the crystal lattice energy was increasing because the crystal lattice breaking process is considered in thermodynamic solubility, but not in the kinetic solubility measurement.

We further investigated which molecular structural parameters, except for lipophilicity, govern the discrepancy between kinetic solubility and thermodynamic solubility in JP2 among the compounds in category (2) since the studies about the factor of the discrepancy between thermodynamic solubility and kinetic solubility have never reported. Several studies have reported the effects of the aromatic ring and the fraction of $s p^{3}$ hybridized carbon atoms on solubility. ${ }^{22,39)}$ First, we explored the relationship between the discrepancy and ratio $s p^{3}\left(\mathrm{R} s p^{3}\right)$, which are as follows: $\mathrm{R} s p^{3}=s p^{3}$ hybridized carbon count $/ s p$ and $s p^{2}$ hybridized carbon count.

The discrepancy between the kinetic solubility and thermodynamic solubility in JP2 tended to increase as the R $s p^{3}$ value decreased (Fig. 8b). A smaller $\mathrm{R} s p^{3}$ value, which indicated that the molecule would be more flat, increased the opportunity for $\pi-\pi$ stacking. Several studies have reported that the hydrogen bond donor and acceptor stabilize the crystal packing and increase the melting point of the molecule. ${ }^{24,40,41)}$ The solubility was increased by reducing the crystal lattice energy, which resulted in a reduced number of strong hydrogen bonds. We evaluated the effect of the hydrogen bond donor and ac- ceptor on the discrepancy between kinetic solubility and thermodynamic solubility. The discrepancy was much larger when the sum of hydrogen bond donor and acceptor count was greater than 8 (Fig. 8c).

Influence of Thermodynamic Solubility on Oral Absorption The effect of thermodynamic solubility on rat oral absorption was investigated. These results are shown in Figs. 9a and $b$. Thermodynamic solubility in JP2 was lower, resulting in poorer absorption. Despite poor thermodynamic solubility in JP2, the compounds with good solubility in JP2-containing GCDC showed sufficient bioavailability. This is due to the bile acid in the gastrointestinal tract, which plays a key role in the solubilization of poorly soluble compounds and enhances oral absorption. ${ }^{42)}$ Thus, it is important to evaluate the aqueous solubility contained in the bile acid for poorly soluble compounds because there is the risk that their absorption is strongly affected by the effect of food. In addition, these results demonstrated that JP2-containing $20 \mathrm{~mm}$ GCDC was suitable to simulate fluid from fed-state intestine fluid. Compared to thermodynamic solubility in JP2, thermodynamic solubility in JP2-containing $20 \mathrm{~mm}$ GCDC have a better relationship with bioavailability because a few bile acids were secreted in rats during the fasting state.

We further investigated the minimum thermodynamic solubility sufficient for oral absorption. The minimum thermodynamic solubility in JP2 and JP2-containing GCDC required
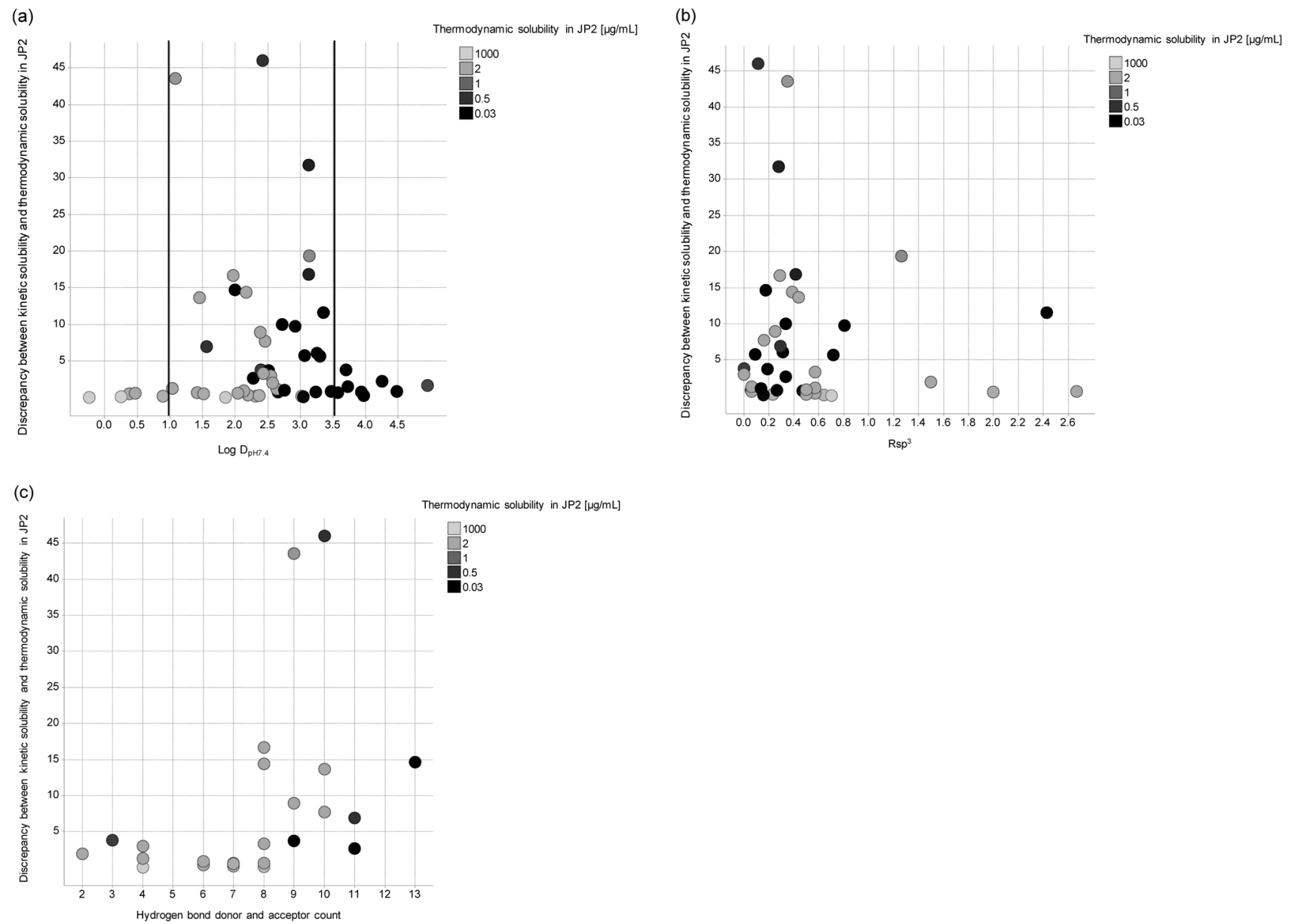

Fig. 8. Discrepancy between the Kinetic Solubility and Thermodynamic Solubility in JP2 as a Function of Measured Log $D_{\mathrm{pH} 7.4}$ (a); R $s p^{3}$ (b); Hydrogen Bond Donor and Acceptor Count (c) 


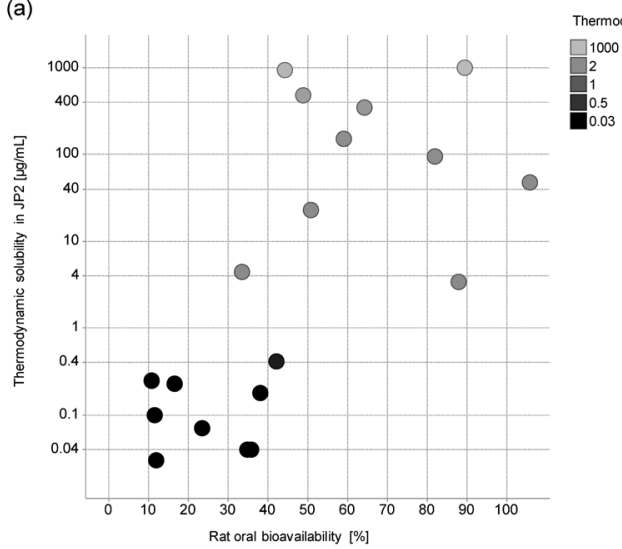

(c)

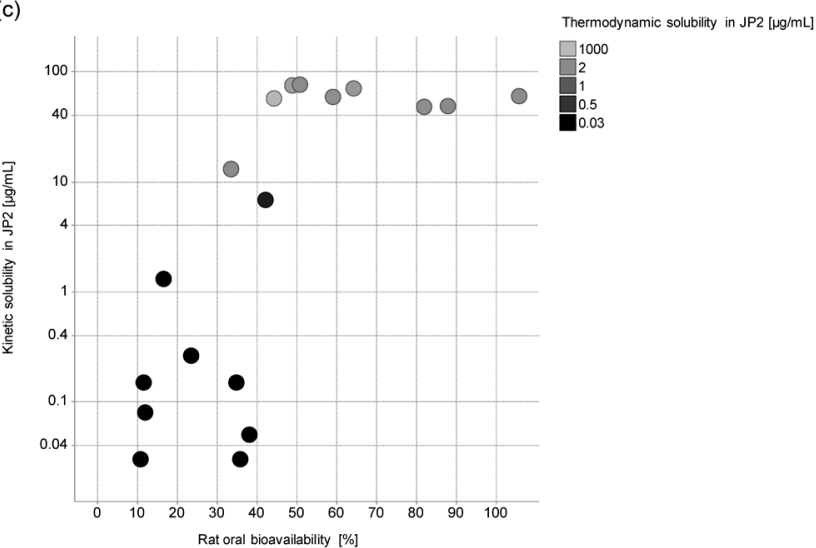

(b)

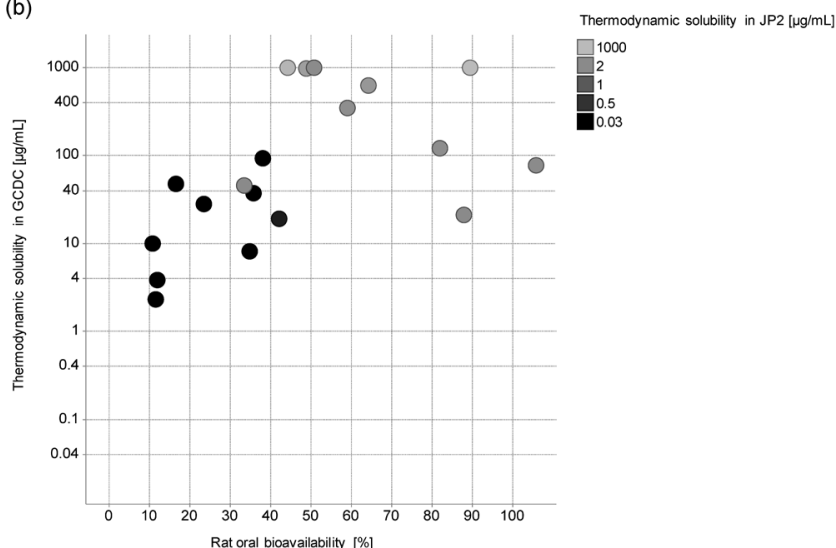

(d)

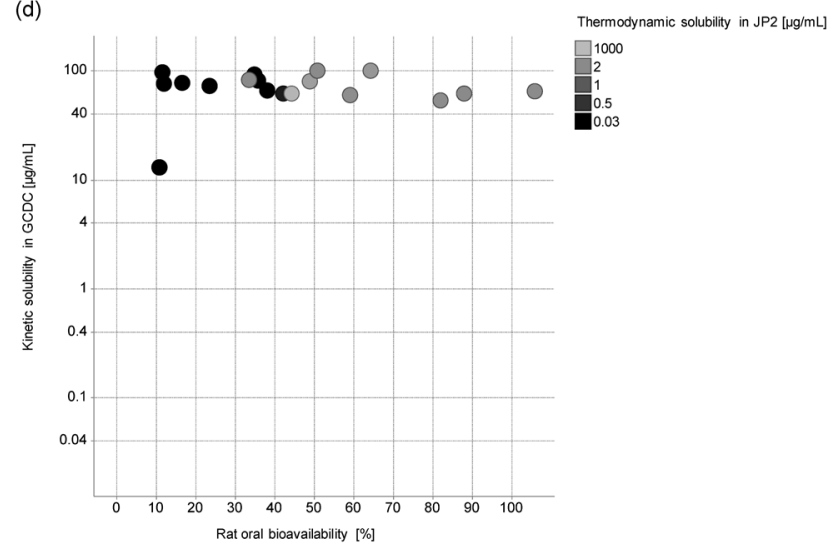

Fig. 9. Correlation between Rat Oral Bioavailability and Thermodynamic Solubility in JP2 (a); Thermodynamic Solubility in JP2-Containing GCDC (b); Kinetic Solubility in JP2 (c); Kinetic Solubility in JP2-Containing GCDC (d)

for acceptable bioavailability $(>30 \%)$ was more than $1 \mu \mathrm{g} / \mathrm{mL}$ and $10 \mu \mathrm{g} / \mathrm{mL}$, respectively. Compared to thermodynamic solubility, kinetic solubility in both the JP2 and JP2-containing GCDC exhibited a poorer correlation with oral bioavailability (Figs. 9c, d). Specifically, kinetic solubility in JP2-containing GCDC showed no correlation with oral bioavailability. The crystal lattice must be broken up and the separate molecules must be solvated in order to be absorbed since the drug suspension in $0.5 \%$ aqueous methylcellulose is administered to rats. Thus, thermodynamic solubility based on the process of crystal lattice break and the solvation of molecules had better correlation with oral absorption than kinetic solubility.

Solubility is an important factor that affects the absorption rate. The MAT is used as a parameter to measure the absorption rate, which is calculated by subtracting mean residence time after i.v. administration from mean residence time after oral administration. In this study, we evaluated the effect of the thermodynamic solubility on the MAT. A higher thermodynamic solubility in both the JP2 and JP2-containing GCDC resulted in a decrease in MAT (Figs. 10a, b). In contrast, MAT was independent of kinetic solubility (Figs. 10c, d).

These results indicated that the dissolution parameter measured in vitro, that is the thermodynamic solubility, was positively correlated with the oral absorption and MAT. In addition, the bioavailability determinations based on the kinetic solubility may be misleading in the lead optimization and candidate selection process. Overall, the thermodynamic solubility is a very useful parameter to predict the oral absorption and provides valuable information for the solid dosage form.

\section{Conclusion}

In this study, we have described the medium throughput approach for physicochemical profiling using a small amount of drug substance, which contributes to the developability assessment of early drug discovery. Our investigation of the relationship between thermodynamic solubility and lipophilicity demonstrated that thermodynamic solubility may be classified into 3 groups according to their $\log D$; a very soluble group is defined as a $\log D$ value below 1, slightly soluble is within the $\log D$ range of 1 to 3.5 and poorly soluble is defined as a $\log D$ value above 3.5. In $\log D$ between 1 and 3.5 , the thermodynamic solubility reflects the sum of the $\log D$, aromatic ring count and the hydrogen bond acceptor and donor count, suggesting that the $\pi-\pi$ stacking and hydrogen bond may be the major interactions that influence crystal packing, which affects thermodynamic solubility. Furthermore, thermodynamic solubility is useful for the criteria of acceptable bioavailability evaluation. Thus, thermodynamic solubility with regard to its solid-state properties as measured by physicochemical profiling enables the rational lead optimization and efficient candidate selection in early drug discovery.

Acknowledgments We would like to appreciate DMPK Research Laboratories and Bio Molecular Research Laboratories, Pharmaceutical Research Division, Takeda Pharmaceutical Company, Ltd. for the conduct of kinetic solubility mea- 
(a)

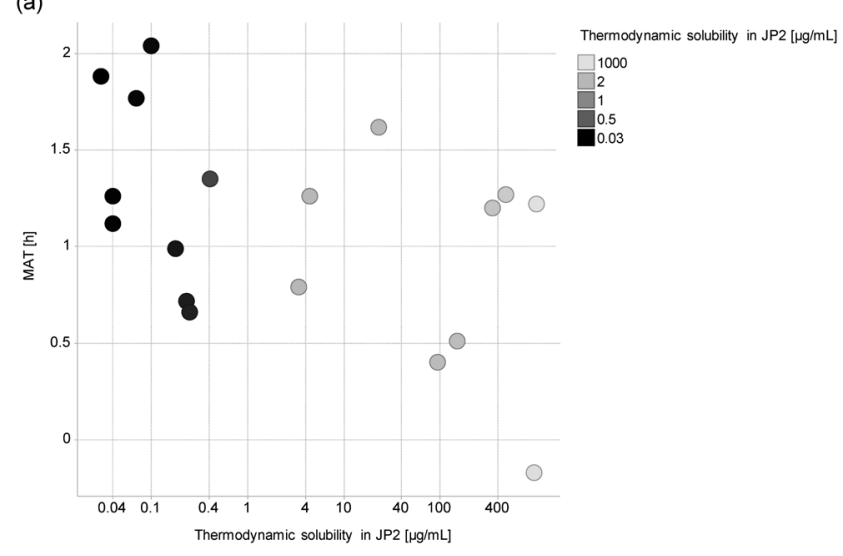

(c)

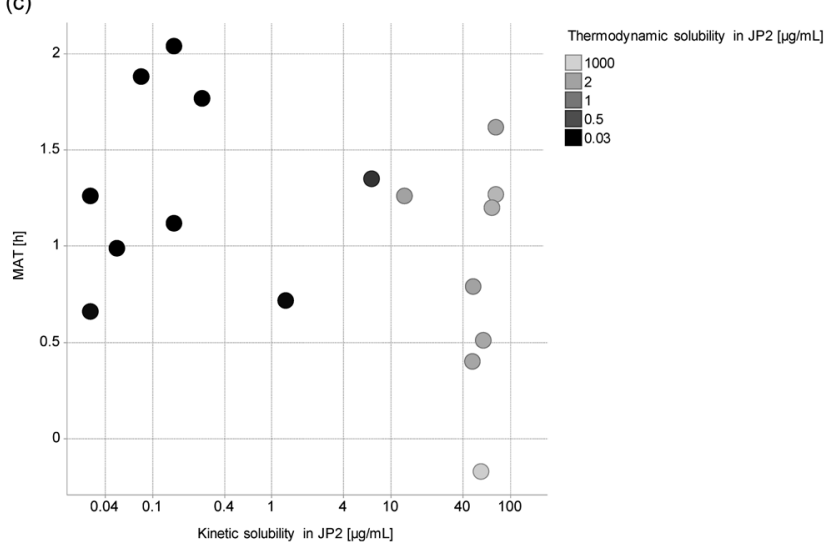

(b)

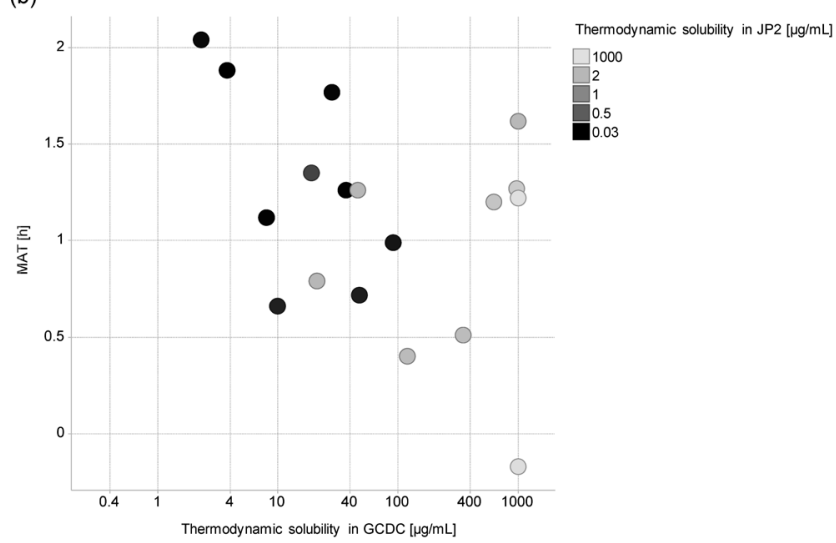

(d)

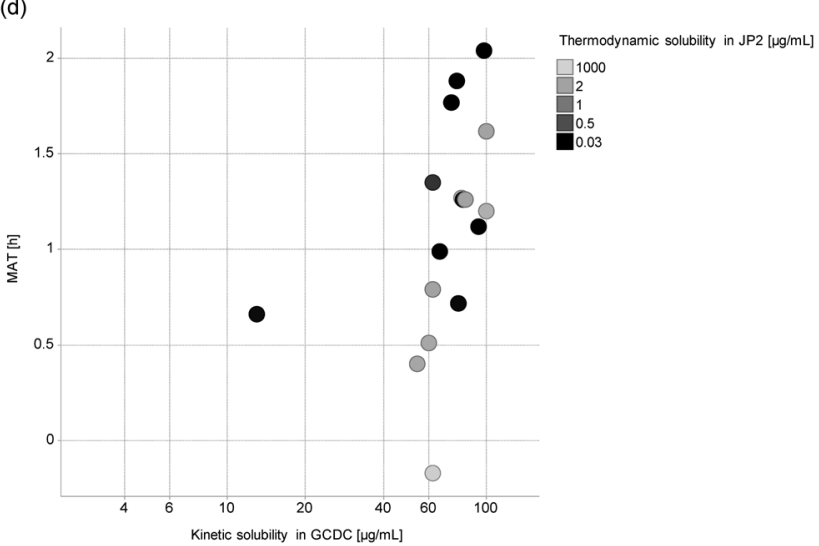

Fig. 10. Correlation between MAT and Thermodynamic Solubility in JP2 (a); Thermodynamic Solubility in JP2-Containing GCDC (b); Kinetic Solubility in JP2 (c); Kinetic Solubility in JP2-Containing GCDC (d)

surement and PK study. Thanks are given to the colleagues of Physicochemistry at Analytical Development Laboratories in CMC Center of Takeda Pharmaceutical Company, Ltd. for their helpful discussions; especially Dr. Takashi Kojima for his crucial reading and comments of this manuscript.

\section{References}

1) Colombo P., Sonvico F., Colombo G., Bettini R., Pharm. Res., 26, 601-611 (2009)

2) Helliwell M., Taylor D., Prof. Nurse, 8, 313-317 (1993).

3) Lipinski C. A., J. Pharmacol. Toxicol. Methods, 44, 235-249 (2000).

4) Stegemann S., Leveiller F., Franchi D., de Jong H., Lindén H., Eur. J. Pharm. Sci., 31, 249-261 (2007).

5) Lipinski C. A., Lombardo F., Dominy B. W., Feeney P. J., $A d v$. Drug Deliv. Rev., 46, 3-26 (2001).

6) Brittain H. G., "Polymorphism in Pharmaceutical Solids," 2nd ed., Marcel Dekker, New York, 1999.

7) Byrn S. R., Pfeiffer R. R., Stowell J. G., "Solid State Chemistry of Drugs," 2nd ed., SSCI Incorporation, West Lafayette, 1999.

8) Haleblian J. K., J. Pharm. Sci., 64, 1269-1288 (1975).

9) Singh D., Marshall P. V., Shields L., York P., J. Pharm. Sci., 87, 655-662 (1998).

10) Pudipeddi M., Serajuddin A. T., J. Pharm. Sci., 94, 929-939 (2005).

11) Lipinski C. A., "Pharmaceutical profiling in drug discovery for lead selection,” ed. by Borchardt R.T., Kerns E.H., Lipinski C.A., Thakker D.R., Wang B., AAPS Press, Arlington, Virginia, 2004, pp. 93-125.

12) Saal C., Petereit A. C., Eur. J. Pharm. Sci., 47, 589-595 (2012). 13) Lipinski C. A., Drug Discov. Today Technol., 1, 337-341 (2004).
14) Congreve M., Carr R., Murray C., Jhoti H., Drug Discov. Today, 8, 876-877 (2003).

15) Didziapetris R., Japertas P., Avdeef A., Petrauskas A., J. Drug Target., 11, 391-406 (2003).

16) Wang J., Hou T., Comb. Chem. High Throughput Screen., 14, 328 338 (2011).

17) Zhang L., Zhu H., Mathiowetz A., Gao H., Bioorg. Med. Chem., 19, 5763-5770 (2011).

18) Ali J., Camilleri P., Brown M. B., Hutt A. J., Kirton S. B., J. Chem. Inf. Model., 52, 420-428 (2012).

19) Ritchie T. J., Macdonald S. J., Young R. J., Pickett S. D., Drug Discov. Today, 16, 164-171 (2011).

20) Thomas V. H., Bhattachar S., Hitchingham L., Zocharski P., Naath M., Surendran N., Stoner C. L., El-Kattan A., Expert Opin. Drug Metab. Toxicol., 2, 591-608 (2006).

21) Wassvik C. M., Holmén A. G., Bergström C. A., Zamora I., Artursson P., Eur. J. Pharm. Sci., 29, 294-305 (2006).

22) Cheng A., Merz K. M. Jr., J. Med. Chem., 46, 3572-3580 (2003).

23) Yang Y., Engkvist O., Llinàs A., Chen H., J. Med. Chem., 55, 3667-3677 (2012)

24) Di L., Fish P. V., Mano T., Drug Discov. Today, 17, 486-495 (2012). 25) Hermans P. H., Weidinger A., J. Appl. Phys., 19, 491-506 (1948).

26) Ikeda Y., Ban J., Ishikawa T., Hashiguchi S., Urayama S., Horibe H., Chem. Pharm. Bull., 56, 1406-1411 (2008).

27) Yamamura S., Momose Y., Int. J. Pharm., 212, 203-212 (2001).

28) Hoelke B., Gieringer S., Arlt M., Saal C., Anal. Chem., 81, 3165 $3172(2009)$.

29) Komura H., Kawahara I., Shigemoto Y., Matsuda K., Ano R., Murayama Y., Moriwaki T., Yoshida N. H., Yakugaku Zasshi, 125, 121-130 (2005). 
30) Masumoto K., Takeyasu A., Oizumi K., Kobayashi T., Yakugaku Zasshi, 115, 213-220 (1995).

31) He K., Qian M., Wong H., Bai S. A., He B., Brogdon B., Grace J. E., Xin B., Wu J., Ren S. X., Zeng H., Deng Y., Graden D. M., Olah T. V., Unger S. E., Luettgen J. M., Knabb R. M., Pinto D. J., Lam P. Y., Duan J., Wexler R. R., Decicco C. P., Christ D. D., Grossman S. J., J. Pharm. Sci., 97, 2568-2580 (2008).

32) Nagilla R., Nord M., McAtee J. J., Jolivette L. J., J. Pharm. Sci., 100, 3862-3874 (2011).

33) Sugaya Y., Yoshiba T., Kajima T., Ishihama Y., Yakugaku Zasshi, 122, 237-246 (2002).

34) Ku M. S., "Oral Controlled Release Formulation Design and Drug Delivery: Theory to Practice," Chap. 4, ed. by Wen H., Park K., John Wiley \& Sons, Inc., Hoboken, NJ, U.S.A., 2010, pp. 47-70.

35) Toke O., Monsey J. D., DeKoster G. T., Tochtrop G. P., Tang C., Cistola D. P., Biochemistry, 45, 727-737 (2006).
36) Guha R., Dexheimer T. S., Kestranek A. N., Jadhav A., Chervenak A. M., Ford M. G., Simeonov A., Roth G. P., Thomas C. J., Bioorg. Med. Chem., 19, 4127-4134 (2011).

37) Hill A. P., Young R. J., Drug Discov. Today, 15, 648-655 (2010).

38) Obata K., Sugano K., Saitoh R., Higashida A., Nabuchi Y., Machida M., Aso Y., Int. J. Pharm., 293, 183-192 (2005).

39) Lovering F., Bikker J., Humblet C., J. Med. Chem., 52, 6752-6756 (2009).

40) Leach A. G., Jones H. D., Cosgrove D. A., Kenny P. W., Ruston L., MacFaul P., Wood J. M., Colclough N., Law B., J. Med. Chem., 49, 6672-6682 (2006).

41) Schultes S., de Graaf C., Berger H., Mayer M., Steffen A., Haaksma E. E. J., de Esch I. J. P., Leursb R., Krämer O., Med. Chem. Commun., 3, 584-591 (2012).

42) Mithani S. D., Bakatselou V., TenHoor C. N., Dressman J. B., Pharm. Res., 13, 163-167 (1996). 